\title{
Helical assemblies of plasmonic nanorods as chiral metamaterials
}

\author{
A. Christofi, ${ }^{a, b}$ N. Stefanou, ${ }^{a}$ G. Gantzounis, ${ }^{c}$ and N. Papanikolaou ${ }^{d}$ \\ ${ }^{a}$ University of Athens, Section of Solid State Physics, Panepistimioupolis, GR-157 84 Athens, \\ Greece \\ ${ }^{b}$ Institute of Materials Science, NCSR "Demokritos", Ag. Paraskevi, GR-153 10 Athens, \\ Greece \\ ${ }^{c}$ Graduate Aeronautical Laboratories (GALCIT) and Department of Applied Physics, \\ California Institute of Technology, Pasadena, California 91125, USA \\ ${ }^{d}$ Institute of Microelectronics, NCSR "Demokritos", Ag. Paraskevi, GR-153 10 Athens, Greece
}

\begin{abstract}
We report on the optical properties of a layer-by-layer structure of silver nanorods, with their axes aligned perpendicular to the $z$ direction and mutually twisted through an angle of $60^{\circ}$ from layer to layer, by means of rigorous full electrodynamic calculations using the layer-multiple-scattering method, properly extended to describe axis-symmetric particles with arbitrary orientation. We analyze the complex photonic band structure of this crystal in conjunction with relevant polarization-resolved transmission spectra of finite slabs of it and explain the nature of the different eigenmodes of the electromagnetic field in the light of group theory. Our results reveal the existence of sizable polarization gaps and demonstrate the occurrence of strong optical activity and circular dichroism, combined with reduced dissipative losses, which make the proposed architecture potentially useful for practical applications as ultrathin circular polarizers and polarization rotators.
\end{abstract}

Keywords: multiple scattering method, complex photonic band structure, particle plasmon modes, chiral metamaterials

\section{INTRODUCTION}

In the context of photonic crystals, three-dimensional (3D) chiral dielectric structures were first proposed as artificial composite media that exhibit strong optical activity. ${ }^{1,2}$ Subsequently, such structures were found to possess sizable omnidirectional photonic band gaps ${ }^{3,4}$ as well as partial band gaps for just one of the two circular polarizations ${ }^{5,6}$ that can give rise to strong circular dichroism..$^{7-9}$ More recently, nanoscale chiral architectures of metallic components were studied in relation to negative-index metamaterials ${ }^{10-13}$ and also because of their huge optical activity which exceeds by several orders of magnitude that of naturally occurring chiral materials, ${ }^{14-17}$ thus opening new perspectives in the design of functional components for miniaturized optical devices.

In the present communication we propose and analyze a layer-by-layer helical assembly of silver nanorods, with circular cross section, stacked along the $z$ direction. In each layer the nanorods are centered at the sites of a square lattice with their axes aligned perpendicular to the $z$ direction and mutually twisted through an angle of $60^{\circ}$ from layer to layer. We investigate the optical response of this crystal by means of rigorous full electrodynamic calculations and analyze the nature of the photonic eigenmodes in the light of group theory. Moreover, we study the change in the polarization state of an electromagnetic (EM) wave transmitted through a finite slab of the crystal and reveal the occurrence of strong optical activity effects for any angle of incidence. It is worth noting that the structure under consideration does not support purely left- and right-circularly polarized (LCP and RCP, respectively) eigenmodes, even at normal incidence, in contrast to mostly studied chiral metamaterials with, e.g., $C_{4}$ symmetry. ${ }^{11,13-15,18}$ In this respect, our results and analysis apply to a broader class of chiral structures of lower symmetry, made of resonant plasmonic components in a helical arrangement, and explain aspects of their optical response to a degree that goes beyond existing interpretation.

Further author information: (Send correspondence to A.C.)

A.C.: E-mail: aristi@ims.demokritos.gr, Telephone: +30 2107276780

Metamaterials VII, edited by Allan D. Boardman, Nigel P. Johnson, Richard W. Ziolkowski, Proc. of SPIE Vol. 8423, 84230A - @ 2012 SPIE - CCC code: 0277-786X/12/\$18 - doi: 10.1117/12.918421 


\section{METHOD OF CALCULATION}

Our calculations are based on the full electrodynamic layer-multiple-scattering (LMS) method, ${ }^{19-21}$ which is ideally suited for stratified structures consisting of successive layers of non-overlapping particles (scatterers) of arbitrary shape, arranged with the same two-dimensional (2D) periodicity in the $x-y$ plane while periodicity in the $z$ direction is not a prerequisite. In the spirit of the multiple-scattering approach, the scattering properties of the entire composite structure are obtained from those of its individual building units. The main idea of the LMS method relies on the combined optimal use of two distinct basis sets: (a) A spherical-wave basis for the description of in-plane multiple scattering, which insures fast convergence for scatterers of not too large size parameter and not too strong deviation from the spherical shape. (b) A plane-wave basis for the description of interlayer multiple scattering, which takes advantage of the common 2D periodicity of the different layers. The method solves Maxwell equations in frequency domain and thus dispersion and absorptive losses in the constituent materials can be readily included. Besides the complex photonic band structure of an infinite crystal, associated with a given crystallographic plane, the LMS method can also provide the reflectance, transmittance and absorbance of a finite slab of the crystal at any angle of incidence and, in this respect, it can describe an actual transmission experiment.

At a first step, in-plane multiple scattering is described in a spherical-wave basis using the scattering $T$ matrix of the individual scatterers and appropriate propagator functions. For particles of cylindrical symmetry, the $T$ matrix is first calculated by the extended-boundary-condition method in a coordinate system defined by taking the $z$ axis along the particle axis and then it is transformed into the actual coordinated system using the relevant transformation matrices for the appropriate Euler rotation angles. ${ }^{22}$ Subsequently, interlayer coupling is fully taken into account by properly combining the transmission and reflection matrices of the component layers, such as the $x-y$ layers of Fig. 1, so as to describe multiple scattering between the layers to any order, in a plane-wave representation defined as follows. We write the component of the wavevector of the incident plane wave parallel to the layers, $\mathbf{q}_{\|}$, as $\mathbf{q}_{\|}=\mathbf{k}_{\|}+\mathbf{g}^{\prime}$, where $\mathbf{k}_{\|}$, the reduced wavevector in the surface Brillouin zone, is a conserved quantity in the scattering process and $\mathbf{g}^{\prime}$ is a certain reciprocal vector of the given $2 \mathrm{D}$ lattice. Therefore, the wavevector of the incident wave has the form $\mathbf{K}_{\mathbf{g}^{\prime}}^{ \pm}=\mathbf{k}_{\|}+\mathbf{g}^{\prime} \pm\left[q^{2}-\left(\mathbf{k}_{\|}+\mathbf{g}^{\prime}\right)^{2}\right]^{1 / 2} \hat{\mathbf{e}}_{z}$, where $q$ is the wavenumber, $\hat{\mathbf{e}}_{z}$ is the unit vector along the $z$ axis and the + or $-\operatorname{sign}$ refers to incidence from $z<0$ or from $z>0$, i.e., a wave propagating towards the positive or negative $z$ direction, respectively. Since $\mathbf{k}_{\|}$and the angular frequency $\omega$ are conserved quantities in the elastic scattering process, the scattered field will consist of a series of plane waves with wavevectors

$$
\mathbf{K}_{\mathbf{g}}^{ \pm}=\mathbf{k}_{\|}+\mathbf{g} \pm\left[q^{2}-\left(\mathbf{k}_{\|}+\mathbf{g}\right)^{2}\right]^{1 / 2} \hat{\mathbf{e}}_{z}, \forall \mathbf{g}
$$

and polarizations along $\hat{\mathbf{e}}_{1}$ and $\hat{\mathbf{e}}_{2}$ (polar and azimuthal unit vectors, respectively, associated with every $\mathbf{K}_{\mathrm{g}}^{ \pm}$). It is worth noting that, though the scattered field consists, in general, of a number of diffracted beams corresponding to different $2 \mathrm{D}$ reciprocal lattice vectors $\mathbf{g}$, only beams for which $\mathbf{K}_{\mathbf{g} z}^{ \pm}$is real constitute propagating waves. When $\left(\mathbf{k}_{\|}+\mathbf{g}\right)^{2}>q^{2}$ we have an evanescent beam and the corresponding unit vectors $\hat{\mathbf{e}}_{1}, \hat{\mathbf{e}}_{2}$ become complex but they are still orthonormal: $\hat{\mathbf{e}}_{p} \cdot \hat{\mathbf{e}}_{p^{\prime}}=\delta_{p p^{\prime}}, p\left(p^{\prime}\right)=1,2$.

The ratio of the transmitted or reflected energy flux to the energy flux associated with the incident wave defines the transmittance or reflectance, respectively, of a multilayer slab. On the other hand, for a 3D crystal consisting of an infinite periodic sequence of layers, stacked along the $z$ direction, applying the Bloch condition for the wave field in the region between two consecutive unit slabs leads to an eigenvalue equation, which gives the $z$ component of the Bloch wave vector, $k_{z}$, for the given $\omega$ and $\mathbf{k}_{\|}$. The eigenvalues $k_{z}\left(\omega, \mathbf{k}_{\|}\right)$, looked upon as functions of real $\omega$, define, for each $\mathbf{k}_{\|}$, lines in the complex $k_{z}$ plane. Taken together they constitute the complex band structure of the infinite crystal associated with the given crystallographic plane. A line of given $\mathbf{k}_{\|}$ may be real (in the sense that $k_{z}$ is real) over certain frequency regions, and be complex (in the sense that $k_{z}$ is complex) for $\omega$ outside these regions. It turns out that, for given $\mathbf{k}_{\|}$and $\omega$, out of the eigenvalues $k_{z}\left(\omega, \mathbf{k}_{\|}\right)$none or, at best, a few are real and the corresponding eigenvectors represent propagating modes of the EM field in the given infinite crystal. The remaining eigenvalues $k_{z}\left(\omega, \mathbf{k}_{\|}\right)$are complex and the corresponding eigenvectors represent evanescent waves. These have an amplitude which increases exponentially in the positive or negative $z$ direction and, unlike the propagating waves, do not exist as physical entities in the infinite crystal. However, they are an essential part of the physical solutions of the EM field in a slab of finite thickness. A region of frequency where propagating waves do not exist, for given $\mathbf{k}_{\|}$, constitutes a frequency gap of the EM field for 


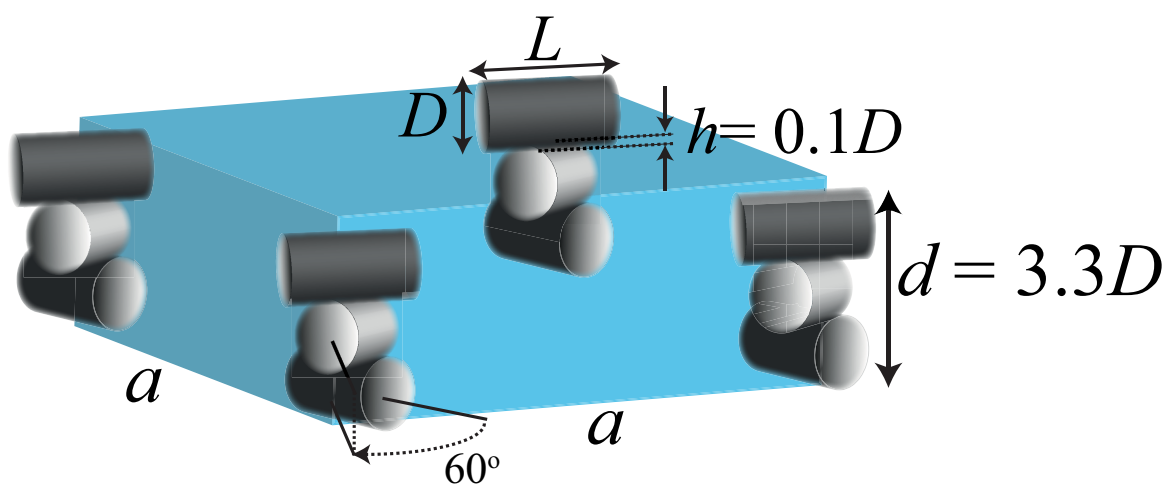

Figure 1. The structure of silver nanorods under consideration: $D=20 \mathrm{~nm}, L=50 \mathrm{~nm}, a=150 \mathrm{~nm}$.

the given $\mathbf{k}_{\|}$. If over a frequency region no propagating wave exists whatever the value of $\mathbf{k}_{\|}$, then this region constitutes an absolute frequency gap.

Adequate convergence of our calculations for the structure under consideration (see Fig. 1) is obtained if we truncate the spherical-wave expansions at $l_{\max }=6$ and take into account $692 \mathrm{D}$ reciprocal lattice vectors $\mathbf{g}$ in the relevant plane-wave expansions, while evaluating the single-particle scattering $T$ matrix with $l_{c u t}=15$ and a Gaussian quadrature integration formula with 1024 points. ${ }^{21,22}$

\section{RESULTS AND DISCUSSION}

We consider a layer-by-layer structure of silver nanorods of length $L=50 \mathrm{~nm}$, with a circular cross section of diameter $D=20 \mathrm{~nm}$, stacked along the $z$ direction. In each layer the nanorods are centered at the sites of a square lattice, of lattice constant $a=150 \mathrm{~nm}$, with their axes aligned in a direction perpendicular to the $z$ axis. The nanorods in consecutive layers are mutually twisted through an angle of $60^{\circ}$ and are separated by a distance $h$, equal to one tenth of their diameter, as shown in Fig. 1. Therefore, the period $d$ of the structure along the $z$ direction comprises three layers $(d=3.3 D)$. With this particular choice of parameters we obtain strong effects with reduced computational effort, though other choices for the twisting angle and interlayer spacing lead to similar results. Obviously, the crystal under consideration lacks invariance under space inversion because of the helical arrangement of the nanorods and thus the appropriate point symmetry group is $D_{2}$, which consists of only proper rotations, and not $D_{2 h}$ that would be if all nanorods were aligned. ${ }^{23}$ For the dielectric function of silver we interpolated to the bulk values measured by Johnson and Christy ${ }^{24}$ that include dissipative losses.

In Fig. 2 we display the complex photonic band structure of the given crystal associated with modes propagating or decaying along the positive $z$ direction. The realistic complex dielectric function of silver causes all bands to become complex in the sense that all values of the wave vector acquire an additional small imaginary part to account for dissipative losses. The bands along the given direction are non-degenerate and have the symmetry of the irreducible representations $(A, B)$ of the $C_{2}$ group, which is a subgroup of $D_{2}{ }^{23}$ i.e., the corresponding eigenmodes are even $(A)$ or odd $(B)$ upon rotation through an angle of $180^{\circ}$ about the $z$ axis. Only the symmetry of the $B$ bands is appropriate for an EM wave incident normally on the (001) surface of the crystal, thus allowing for light transmission. It is worth noting that the $C_{2}$ symmetry does not allow for the existence of LCP and RCP eigenmodes as it would be the case under, e.g., the fourfold rotation $\left(C_{4}\right)$ symmetry. ${ }^{23}$ In our case, each optically active $B$ band has a different degree of LCP and RCP admixture that varies along the band as shown in Fig. 2 . Similar dispersion diagrams are obtained along other directions as well. A careful analysis of the complex band structure of Fig. 2 reveals the existence of (a): two extended bands, one of LCP and one of RCP predominant character, associated with wave propagation in an underlying effective medium with very low dissipative losses and (b): three relatively narrow bands, i.e., as many as the number of nanorods per primitive cell, originating from the fundamental longitudinal dipole-like plasmon modes of the individual nanorods at $2.5 \mathrm{eV},{ }^{25}$ weakly interacting between them. The lowest of these narrow bands, below $2.3 \mathrm{eV}$, has a mixed LCP-RCP character and its imaginary part increases abruptly at low frequencies. The other two narrow bands, one of LCP and one 

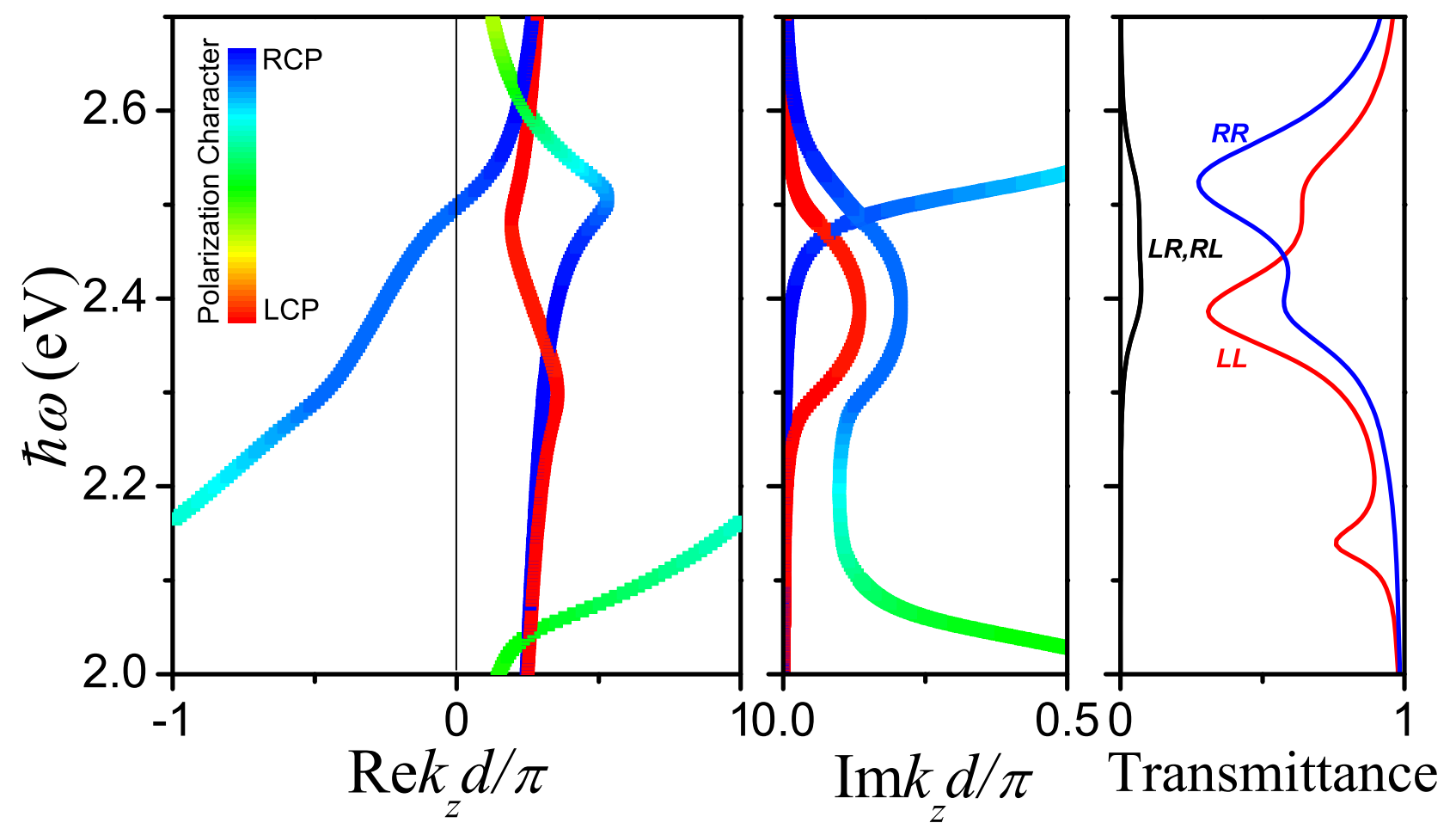

Figure 2. The complex photonic band structure associated with modes propagating or decaying along the positive $z$ direction of the crystal of Fig. 1 and the corresponding transmission spectra associated with different circular polarizations channels at normal incidence on a (001) unit slab of the crystal, three-layers thick.

of RCP predominant character about $2.4 \mathrm{eV}$ and $2.5 \mathrm{eV}$, respectively, interact strongly with the corresponding effective-medium bands leading to the occurrence of considerable hybridization gaps for a specific polarization. In these gap regions, the imaginary part of the corresponding complex bands takes relatively large values. Along an arbitrary direction of the Brillouin zone, all bands retain their predominant circular-polarization character and thus the band diagram keeps its main characteristics.

The right-hand diagram of Fig. 2 shows the transmission properties of a (001) unit slab of the crystal of Fig. 1 consisting of three layers of nanorods, at normal incidence. It can be seen that the transmission spectra associated with the LCP and RCP channels exhibit pronounced differences while the occurrence of cross coupling transmission from LCP to RCP and vice versa implies a non-negligible circular polarization conversion in the range from $2.3 \mathrm{eV}$ to $2.6 \mathrm{eV}$. Over the frequency range of the polarization gaps only incident waves of opposite handedness are allowed to pass through. Such regions of polarization-selective transmission have also been encountered in metallic helix arrays. ${ }^{12,16}$

The polarization state of a wave transmitted through a finite slab of the crystal under consideration is directly obtained from the corresponding transmission matrix. It is worth noting that, in the frequency region of interest, we are below the diffraction limit for light incident on the (001) surface of the crystal at any angle, as appropriate for the metamaterial regime. Therefore, only the zero-order diffraction channel yields a propagating beam and the (electric-field) amplitude of the transmitted wave is obtained from that of the incident wave through a $2 \times 2$ complex transmission matrix, $\mathbf{t}$, as follows $E_{p}^{t r}=\sum_{p^{\prime}} t_{p p^{\prime}} E_{p^{\prime}}^{\text {in }}$ where $p\left(p^{\prime}\right)=1,2$ refers to the linearly polarized waves with the electric field oscillating in or perpendicular to the plane of incidence, respectively, in accordance with the plane-wave basis employed in the LMS method (see Sec. 2). We note that at normal incidence on the (001) surface of the crystal, these polarization directions coincide with the $x$ and $y$ axes, respectively. One can easily switch from the basis of linearly polarized waves $\left[p\left(p^{\prime}\right)=1,2\right]$ to the basis of circularly polarized waves 


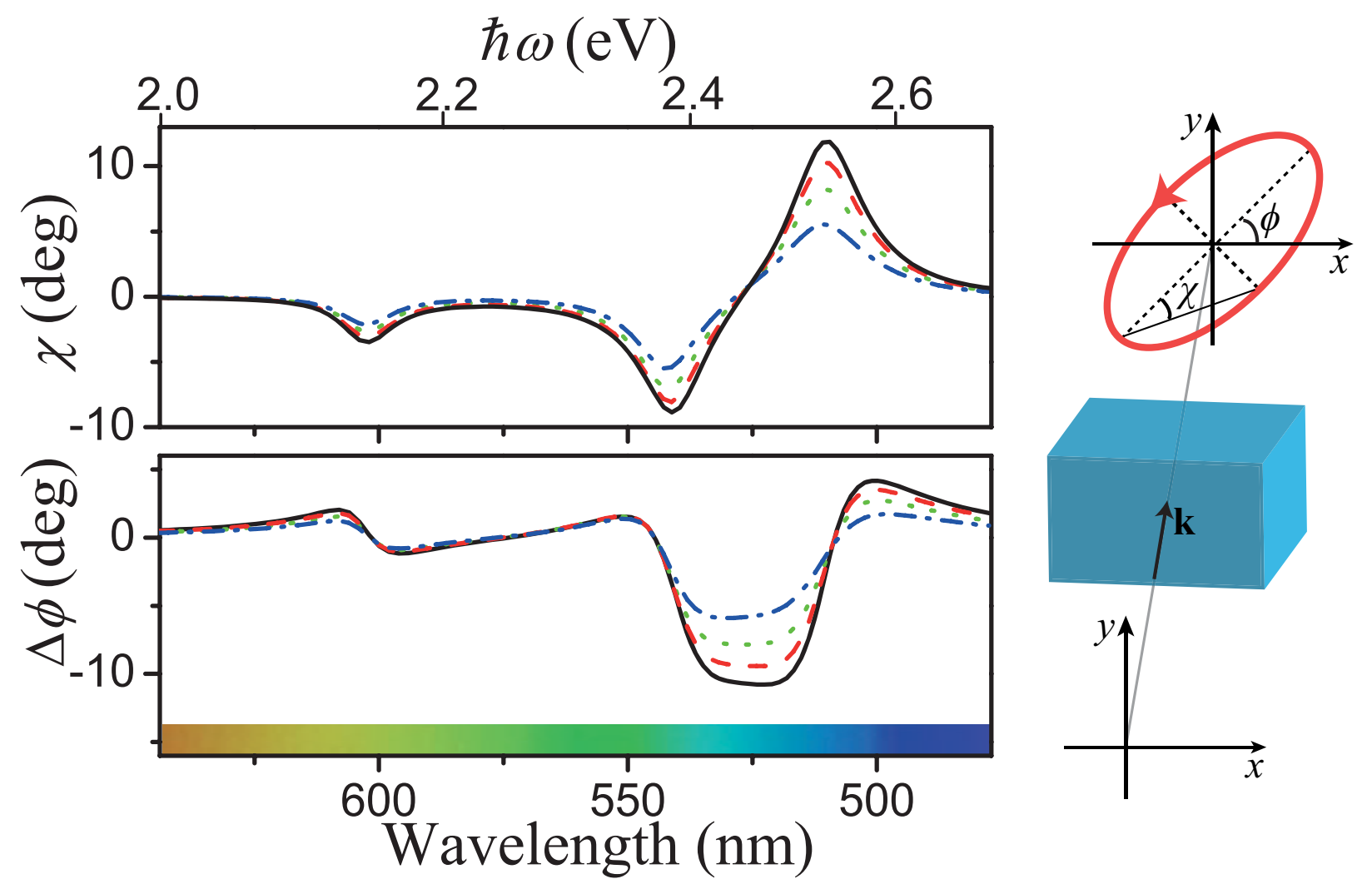

Figure 3. Variation of the polarization-averaged ellipticity (upper diagram) and azimuth rotation (lower diagram) angles of a wave transmitted through a (001) unit slab of the crystal of Fig. 1, three-layers thick, for different angles of incidence: $0^{\circ}$ (solid line), $30^{\circ}$ (dashed line), $45^{\circ}$ (dotted line), 60 (dashed-dotted line) in the visible spectrum.

$\left[p\left(p^{\prime}\right)=L, R\right.$, for LCP and RCP states, respectively] with the help of a similarity transformation

$$
\left(\begin{array}{cc}
t_{L L} & t_{L R} \\
t_{R L} & t_{R R}
\end{array}\right)=\frac{1}{\sqrt{2}}\left(\begin{array}{cc}
1 & -i \\
1 & i
\end{array}\right)\left(\begin{array}{cc}
t_{11} & t_{12} \\
t_{21} & t_{22}
\end{array}\right)\left[\frac{1}{\sqrt{2}}\left(\begin{array}{cc}
1 & -i \\
1 & i
\end{array}\right)\right]^{-1}
$$

An incoming plane wave of amplitude $E_{0}$, propagating along the positive $z$ direction, linearly polarized at an angle $\phi_{0}$ with respect to the $x$ axis, can be decomposed into LCP and RCP waves of amplitudes $E_{L}^{i n}=$ $E_{0} \exp \left(-i \phi_{0}\right) / \sqrt{2}$ and $E_{R}^{i n}=E_{0} \exp \left(i \phi_{0}\right) / \sqrt{2}$, respectively. The corresponding transmitted fields have amplitudes $E_{L}^{t r}=\frac{E_{0}}{\sqrt{2}}\left[t_{L L} \exp \left(-i \phi_{0}\right)+t_{L R} \exp \left(i \phi_{0}\right)\right]$ and $E_{R}^{t r}=\frac{E_{0}}{\sqrt{2}}\left[t_{R L} \exp \left(-i \phi_{0}\right)+t_{R R} \exp \left(i \phi_{0}\right)\right]$ which correspond to an in general elliptically polarized wave with the long axis of the ellipse forming an angle

$$
\phi=\frac{1}{2}\left[\arg \left(E_{R}^{t r}\right)-\arg \left(E_{L}^{t r}\right)\right]
$$

with the $x$ axis and with ellipticity angle

$$
\chi=\arctan \frac{\left|E_{R}^{t r}\right|-\left|E_{L}^{t r}\right|}{\left|E_{R}^{t r}\right|+\left|E_{L}^{t r}\right|},
$$

as shown in Fig. 3. We adopt the polarization azimuth rotation angle, $\Delta \phi=\phi-\phi_{0}$ as a measure of circular birefringence and the ellipticity angle $\chi$ as a measure of circular dichroism.

In Fig. 3 we depict the calculated polarization-averaged ellipticity and azimuth rotation angles of a wave transmitted through a (001) unit slab of the structure under consideration, three-layers thick, for different angles of incidence. The polarization-averaged ellipticity angle vanishes at $\hbar \omega=2.44 \mathrm{eV}$, for all different angles of 
incidence, which indicates a pure optical activity effect. At this frequency, the polarization-averaged azimuth rotation of the transmitted wave varies from $-11^{\circ}$ (at normal incidence) to $-6^{\circ}$ (for incidence at an angle of $60^{\circ}$ ). In terms of rotatory power per sample thickness equal to one wavelength, the optical activity of the considered three-layers slab can be as large as $-88^{\circ}$ which is an order of magnitude larger than that of naturally occurring optically active materials and comparable with that of other chiral metamaterials. ${ }^{11}$ Stronger optical activity effects are obtained for thicker slabs, while the spectral response can be tuned in a controllable manner by choosing the rod length so as to appropriately shift its plasmon resonance. ${ }^{25}$ Therefore, multilayer slabs of the given crystal provide a huge polarization-averaged optical activity for any angle of incidence, with quite low transmission losses (typically a few $\mathrm{dB}$ ), and thus they can be useful for practical applications as ultrathin circular polarizers and polarization rotators.

\section{CONCLUSION}

In summary, we reported a comprehensive study of the optical properties of a 3D helical structure of silver nanorods by means of rigorous full electrodynamic calculations using the LMS method, properly extended to describe axis-symmetric scatterers with arbitrary orientation. We analyzed the complex photonic band structure of the metamaterial under consideration in conjunction with relevant polarization-resolved transmission spectra and explained the nature of the different eigenmodes in the light of group theory. We showed that multilayer slabs of the crystal exhibit frequency-tunable strong optical activity and circular dichroism with relatively low dissipative losses.

\section{ACKNOWLEDGMENTS}

A. Christofi is supported by NCSR "Demokritos" through a postgraduate fellowship.

\section{REFERENCES}

1. V. Karathanos, N. Stefanou, and A. Modinos, "Optical activity of photonic crystals," J. Mod. Opt. 42, 619-626 (1995).

2. I. Hodgkinson, Q. H. Wu, B. Knight, A. Lakhtakia, and K. Robbie, "Vacuum deposition of chiral sculptured thin films with high optical activity," Appl. Opt. 39, 642-649 (2000).

3. A. Chutinan and S. Noda, "Spiral three-dimensional photonic-band-gap structure," Phys. Rev. B 57, R2006-R2008 (1998).

4. O. Toader and S. John, "Proposed square spiral microfabrication architecture for large three-dimensional photonic band gap crystals," Science 292, 1133-1135 (2001).

5. J. Lee and C. Chan, "Polarization gaps in spiral photonic crystals," Opt. Express 13, 8083-8088 (2005).

6. M. Thiel, M. Decker, M. Deubel, M. Wegener, S. Linden, and G. von Freymann, "Polarization stop bands in chiral polymeric three-dimensional photonic crystals," Adv. Mater. 19, 207-210 (2007).

7. P. C. P. Hrudey, B. Szeto, M. J. Brett, "Strong circular Bragg phenomena in self-ordered porous helical nanorod arrays of Alqu," Appl. Phys. Lett. 88, 251106 (2006).

8. S. Furumi and Y. Sakka, "Chiroptical properties induced in chiral photonic-bandgap liquid crystals leading to a highly efficient laser-feedback effect," Adv. Mater. 18, 775-780 (2006).

9. M. Mitov and N. Dessaud, "Going beyond the reflectance limit of cholesteric liquid crystals," Nat. Mater. 5, 361-364 (2006).

10. J. B. Pendry, "A chiral route to negative refraction," Science 306, 1353-1355 (2004).

11. E. Plum, J. Zhou, J. Dong, V. A. Fedotov, T. Koschny, C. M. Soukoulis, and N. I. Zheludev, "Metamaterial with negative index due to chirality," Phys. Rev. B 79, 035407 (2009).

12. C. Wu, H. Li, Z. Wei, X. Yu, and C. T. Chan, "Theory and experimental realization of negative refraction in a metallic helix array," Phys. Rev. Lett. 105, 247401 (2010).

13. R. Zhao, L. Zhang, J. Zhou, Th. Koschny, and C. M. Soukoulis, "Conjugated gammadion chiral metamaterial with uniaxial optical activity and negative refractive index," Phys. Rev. B 83, 035105 (2011).

14. A. Papakostas, A. Potts, D. M. Bagnall, S. L. Prosvirnin, H. J. Coles, and N. I. Zheludev, "Optical manifestations of planar chirality," Phys. Rev. Lett. 90, 107404 (2003). 
15. E. Plum, V. A. Fedotov, A. S. Schwanecke, N. I. Zheludev, and Y. Chen, "Giant optical gyrotropy due to electromagnetic coupling," Appl. Phys. Lett. 90, 223113 (2007).

16. J. K. Gansel, M. Thiel, M. S. Rill, M. Decker, K. Bade, V. Saile, G. von Freymann, S. Linden, and M. Wegener, "Gold helix photonic metamaterial as broadband circular polarizer," Science 325, 1513-1515 (2009).

17. A. Guerrero-Martinez, J. L. Alonso-Gomez, B. Auguie, M. M. Cid, L. M. Liz-Marzan, "From individual to collective chirality in metal nanoparticles," Nano Today 6, 381-400 (2011).

18. A. Andryieuski, C. Menzel, C. Rockstuhl, R. Malureanu, F. Lederer, and A. Lavrinenko, "Homogenization of resonant chiral metamaterials," Phys. Rev. B 82, 235107 (2010).

19. N. Stefanou, V. Yannopapas, and A. Modinos, "Heterostructures of photonic crystals: frequency bands and transmission coefficients," Comput. Phys. Commun. 113, 49-77 (1998).

20. N. Stefanou, V. Yannopapas, and A. Modinos, "MULTEM2: a new version of the program for transmission and band-structure calculations of photonic crystals," Comput. Phys. Commun. 132, 189-196 (2000).

21. G. Gantzounis and N. Stefanou, "Layer-multiple-scattering method for photonic crystals of nonspherical particles," Phys. Rev. B 73, 035115 (2006).

22. M. I. Mishchenko, L. D. Travis, and A. A. Lacis, [Scattering, Absorption, and Emission of Light by Small Particles], Cambridge University Press, Cambridge, U.K. (2002).

23. T. Inui, Y. Tanabe, and Y. Onodera, [Group Theory and its Applications in Physics], Springer, Berlin (1990).

24. P. B. Johnson and R. W. Christy, "Optical constants of the noble metals," Phys. Rev. B 6, 4370-4379 (1972).

25. C. Tserkezis, N. Papanikolaou, E. Almpanis, and N. Stefanou, "Tailoring plasmons with metallic nanorod arrays," Phys. Rev. B 80, 125124 (2009). 